RESEARCH ETHICS

\title{
Children's understanding of the risks and benefits associated with research
}

\author{
T M Burke, R Abramovitch, S Zlotkin
}

J Med Ethics 2005;31:715-720. doi: 10.1136/jme.2003.003228

See end of article for authors' affiliations .....................

Correspondence to: Tara M Burke, Department of Psychology, Ryerson University, 350 Victoria Street, Toronto, Ontario, M5B 2K3, Canada; tburke@ryerson.ca

Received 21 January 2003 In revised form 1 February 2005

Accepted for publication 19 February 2005

\begin{abstract}
Objective: The objective of the current study was to maximise the amount of information children and adolescents understand about the risks and benefits associated with participation in a biomedical research study.

Design: Participants were presented with one of six hypothetical research protocols describing how to fix a fractured thigh using either a "standard" cast or "new" pins procedure. Risks and benefits associated with each of the treatment options were manipulated so that for each one of the six protocols there was either a correct or ambiguous choice.

Participants and sefting: Two hundred and fifty one children, ages 6-15 (53\% boys), and 237 adults (30\% men) were interviewed while waiting for a clinic appointment at the Hospital for Sick Children.

Results: Using standardised procedures and questionnaires, it was determined that most participants, regardless of age group, were able to understand the basic purpose and procedures involved in the research, and most were able to choose the "correct" operation. The younger children, however, showed an overall preference for a cast operation, whereas the older participants were more likely to choose the pins. Conclusions: By creating age appropriate modules of information, children as young as six years can understand potentially difficult and complex concepts such as the risks and benefits associated with participation in biomedical research. It appears, however, that different criteria were used for treatment preference, regardless of associated risks; older participants tended to opt for mobility (the pins procedure) whereas younger participants stayed with the more familiar cast operation.
\end{abstract}

$\mathrm{R}$ esearch involving children is essential for advancements in paediatric medicine, and development of age appropriate methods of informing children prior to obtaining their agreement to participate in research is of vital importance because informed consent is at the core of ethical research. Actually achieving informed consent poses many problems even with adults, but it is especially difficult with research that involves children, as it is assumed that children are not legally competent to consent for themselves. Indeed, the term consent is not generally considered appropriate for minors, so their agreement to participate is now generally referred to as "assent" (or "dissent" if they refuse). Whereas the term "consent" has both legal and ethical implications, assent is based entirely on ethical concerns. Consent assumes that the individual (adult) is fully cognisant of all aspects of the research that they (or their child) are being invited to participate in; this must be granted before the research proceeds. The notion of assent, however, recognises that even young children, who may lack the cognitive capacity to make a truly informed decision, can still be given the chance to be part of the decision making process. ${ }^{1}$ The requirement for the assent of the child participating in research is widely accepted in Canada ${ }^{2}$ and internationally ${ }^{4}$ and is based on the ethical principle of respect for the child. ${ }^{3}$ If a child's assent is to be meaningful, however, he/she must understand the nature of the research, including information about procedures and potential benefits and harms, before a decision can be made (along with parental authorisation) about whether to agree to participate. Further, participation in research does not typically lead to direct benefits for the participants as might consent to treatment; although the knowledge gained from the research may eventually prove invaluable and help many others, this may not be an easy distinction for any participant, regardless of age, to make. ${ }^{5}$
Although the disclosure process is ideally an interactive dialogue, where the prospective participant is invited to ask questions and discuss the details of the research with the investigators and others as necessary, the written consent document is an important starting point to ensure that most of the relevant information is presented in a neutral manner. If written forms for children are to become common practice, however, research is required to examine the usefulness of the forms, and to develop the type of language that is most readily understood by the youngest children who are asked to give assent, so they may more fully take part in the decision making process.

A search of the literature reveals only a handful of studies examining children's ability to understand information about participation in clinical research. Weithorn and Campbell used hypothetical medical scenarios to examine the competence of 9, 14, 18 and 21 year olds to consent to treatment. They found that at 14 years of age the children demonstrated a level of competency equivalent to that of adults. At nine years of age children were able to make a "reasonable" choice, but were less able to understand and consider many of the critical elements of the treatment information in making their choice. ${ }^{6}$ Others have found that among six year olds, the aspect of informed consent least understood was the idea that future benefits might result from an immediate "cost". ${ }^{7}$ Susman et $a l^{8}$ scored understanding of the procedure as adequate if $50 \%$ of the procedures were named by the participants. This approach is not satisfactory, however, as it does not account for the fact that some elements of the information may be considered more important than others. While young children may be able to understand what is being asked of them in a research study in terms of their actual participation, they may have difficulty following concepts such as confidentiality and voluntariness. ${ }^{9}$ 
Ondrusek et $a l^{10}$ found-for example-that children under the age of nine, who were asked to give assent to a clinical trial, poorly understood most of the associated concepts, including procedure and voluntariness. They suggest that the typical age for assent should perhaps be increased from seven to nine, assuming that the lack of understanding was a function of developmental ability rather than how the material was presented. Generally, it appears that the extent of information understood seems to vary from study to study. There has, however, been little in the way of systematic research that focuses on the different components of the consent/assent process.

The present study focuses on children's ability to understand varying risks and benefits associated with their participating in a research study. Among the different kinds of information disclosed to participants or patients, the risks of a therapeutic or research procedure are often the least well understood, regardless of age. Among 200 adult cancer patients questioned within one day of signing consent forms for chemotherapy, radiation, or surgery, only $60 \%$ understood the purpose and nature of the procedure, and only 55\% correctly listed even one major risk. ${ }^{11}$ Similarly, in two separate studies, adult participants in a clinical trial, and patients questioned after surgery, were tested for understanding. Only 20 and $23 \%$ of individuals, respectively, appeared to be adequately informed about the risks of the procedures. ${ }^{12}{ }^{13}$ Commenting on the possible interpretation of these results, Silva and Sorrel ${ }^{14}$ suggest that one reason patients may have scored poorly on understanding or recall of the risks is that this information was played down in the clinical information forms. Further, Abramovitch et $a l^{15}$ found that children often have difficulty in understanding the risks and benefits of psychological studies.

The first step in disclosing information is to create information forms that are clear and appropriately worded for the target population. Previous research has found that, in terms of readability, such forms are generally written at an inappropriate level for those for whom they are intended. ${ }^{16}$ The problem, of course, is that if participants are unable to comprehend the information presented to them, they are unlikely to be truly "informed". Thus the ethical and legal requirement of informed consent may often not be met. Forms meant to be understood by children aged 6 to 12 years (generally those in the fifth or sixth grade) are often written at a grade 10 or 11 reading level. ${ }^{17}$ It may also be the case that the length of the information forms is a factor. One study provided participants with information forms that explained a mock experiment comparing a painkiller and a placebo. Each form contained the same basic information, but differed in the amount of descriptive material and therefore in length (short, medium, long). Participants were tested on their understanding of the study and various factors such as the benefits and risks, including what underlying health conditions might put them at high risk for side effects. Understanding was best among those who read the short form; those who read the long form scored the worst. In that study, it was noted that those who read the long form were more likely to falsely label themselves at high or low risk for side effects. ${ }^{17}$

The goal of the current study was to evaluate children's understanding of the risks and benefits associated with participation in a biomedical research study. This study is part of a larger effort to develop information templates, which explain the varying benefit to the participant, varying risk or discomfort to the participant, various research designs, and varying diseases or clinical specific situations. Our goal is to produce a series of standardised, understandable modules describing specific aspects of research protocols, which can be used by researchers across Canada in the development of their specific information forms. There is a potential problem with this type of study, as the general understanding afforded by the assent forms may be confounded by the participant's own experience with and knowledge about a particular condition. ${ }^{10}$ It is possible that pre-existing knowledge on the part of the participants could lead to an inflation in apparent "understanding" of the assent forms that has little to do with how they are written. Participants selected for this study were interviewed regarding their understanding of medical research forms for a condition that they themselves did not suffer from. We felt this approach was necessary to standardise the items included in the forms and isolate the effects of the form from other contributions made by the investigator, the setting, or the patient's illness. The information forms in the present study described two different procedures for treating a fracture: the standard "cast" operation, and the experimental "pins" treatment. Although the procedures were kept constant across conditions, we manipulated the description of the operations so that some had more "good" things associated with them, and others had more "bad" things associated with them, in order to focus on participants' understanding of risks and benefits. Although participants were asked, in the course of the study, which treatment they would prefer, if they had a choice, the focus of the study was on their understanding of the risks and benefits, rather than on their assenting to a particular type of treatment. There were three different good and three different bad things listed for each of the procedures. In some, it appeared that the standard cast operation would be preferable to the new pins procedure, and in some, it seemed the pins were a better option. Both parents and children were interviewed, in order to compare levels of understanding between these two groups.

\section{METHOD}

\section{Participants}

Two hundred and fifty one children, ages 6-15 (53\% boys), and 237 adults (30\% men) were interviewed while waiting for an appointment in one of the clinics at the Hospital for Sick Children. Parents were given a detailed consent form, and children were asked to give their assent to participation in our study. Assent forms for the children were based on the consent forms given to their parents, though in simplified language.

\section{Procedure}

Two interviewers approached parents/care givers and their children in clinic waiting rooms. Both the children and the adults were informed that, if they agreed to be in the study, they would be told about a hypothetical research study and then asked some questions regarding their understanding of what they had been told. Children were told they would be asked to imagine that they themselves were being treated for a medical condition that we would describe, while adults were told they would be asked to imagine that their child was being treated for the condition. It was emphasised that they were not being tested for how well they could remember the information and that they were free to refer to the information sheet describing the studies throughout. They were also told that we were not looking for right or wrong answers, but rather that we wanted to know how clearly the information forms described and explained the research. They were informed that all information collected would remain confidential, that they were free to discontinue their participation at any time, and that they could refuse to answer any questions that made them feel uncomfortable. Once consent and assent were obtained; the participants were randomly assigned to receive one of six information forms (see below). 
One interviewer then read the information forms to the child, while the other interviewer read the form to the adult in a separate area of the waiting room. All individuals, regardless of their age or reading ability, had the information form read to them in order to standardise the procedure. Once the interviewer finished reading the information forms, the participants were asked a series of questions regarding what they had just heard. This standardised semistructured interview was designed to assess both the child's and the adult's understanding of several general categories of information contained in the information form. As appropriate, participants were probed about various aspects of the study. Responses were recorded verbatim.

\section{Information forms}

Information forms were developed that described each research design in the simplest and clearest possible terms (see Appendix A for a copy of one of the six information forms). Each information form was divided into five general parts. These sections were entitled: 1) What is research? 2) Why are we doing this study? 3) What will happen during the study? 4) Are there good things about the study? 5) Are there bad things about the study? A page describing the procedure for the cast and the pin operations was also included after the third section; this page contained a sketch of how the cast and the pins would look on a child's leg. All participants read the same description of the research, its purpose, and the procedures involved.

The manipulation of the risks and benefits took the form of a chart that compared the good things about the cast to the good things about the pins, and the bad things about the cast to the bad things about the pins. There were six different risk/benefit conditions, where the "good things" were either very good, or not particularly good, and where the "bad things" were either very bad, or not too bad. For two of the conditions, the cast was the "correct" choice, for two conditions the pins were the "correct choice", and for the last two conditions, the choice was even-for example, if the cast operation was described as having very "high" good things and very "high" bad things, while the pins were described as having "low" good things, but "high bad things (HHLH), then the "correct" choice would be the cast operation.

\section{Coding}

A coding scheme was developed, based on our pilot studies, which corresponded to the different sections presented in the information form.

\section{Research}

Credit was given if the answer corresponded to one of the reasonable responses associated with the question, based on the statement presented in the information form.

\section{Purpose}

Answers were scored in terms of whether or not the participant was able to give at least one reasonable response. A reasonable response involved either a paraphrase of what was in the information form or a reasonable inference based on the information.

\section{Procedures}

Factual response categories were developed from the information that was presented in the information form. In each study we identified a priori the most crucial procedural element and noted whether it was mentioned.

\section{Risks and benefits}

Answers were scored in terms of whether or not the child was able to give at least one reasonable response, involving either a paraphrase of what was in the information form or a reasonable inference. In addition, participants were asked to indicate, based on the information presented to them, which operation they would choose, if given the opportunity to decide. Therefore a "right" or "wrong" answer was scored, based on the particular combination of good/bad things associated with their assigned condition.

\section{RESULTS}

As many of the questions asked involved multiple or open ended responses, results were generally reported in percentages. No sex differences were found, so data were collapsed across gender. The general categories of information are also collapsed across condition, as all information was identical for all participants, except for the risks and benefits described.

\section{Research}

When asked to explain the meaning of the term "research", a significant age effect was found $\left(X^{2}(3)=66.3, p<0.0001\right)$. Only $24 \%$ of the youngest children (6-9 years) were able to define research, compared to $45 \%$ of the 10 to 12 year olds, and $65 \%$ of the children over the age of 13 years. In contrast, $72 \%$ of the adults were able to provide a reasonable response to this question. Reasonable responses included "Trying new and different ways of doing things to see what works best" or "asking people/children how they feel about treatment".

\section{Purpose}

Although most participants seemed to understand the purpose of the hypothetical studies, a significant difference was found among the age groups when asked: "why are we doing this study?" $\left(X^{2}(3)=18.6, p<0.0003\right)$. Seventy three per cent of the youngest, $87 \%$ of the middle, and $93 \%$ of the oldest children, and $90 \%$ of the adults, gave at least one reasonable response, such as "finding out which operation is better", "finding out which treatment kids prefer", or "helping child/people with broken bones".

\section{General procedure}

No differences were found among the age groups when asked: "what will the doctors and nurses do if $\mathrm{I} / \mathrm{my}$ child decides to be in the study?" $\left(X^{2}(3)=8.9, p>0.05\right)$. Sixty two per cent of the youngest, $65 \%$ of the middle, $71 \%$ of the oldest children, and $76 \%$ of the adults gave at least one reasonable response, such as "will get an operation", "will have some tests-that is, $x$ rays".

\section{Cast procedure}

When asked: "tell me what would happen to you/your child if you/he/she had the 'cast' operation", a significant age effect was found $\left(X^{2}(3)=21.4, p<0.0001\right)$. Eighty per cent of the youngest, $92 \%$ of the middle, and $95 \%$ of the oldest children gave a reasonable response. Ninety six per cent of the adults were also able to answer the question appropriately. Reasonable responses included "I would get a body cast" or "I will need crutches".

\section{Pins procedure}

When asked: "tell me what would happen to you/your child if you/he/she had the "pins" operation, a significant age effect was found $\left(X^{2}(3)=18.4, p<0.0003\right)$. Although $78 \%$ of the youngest gave a reasonable response, over $90 \%$ of individuals in the middle, oldest, and adult categories $(90 \%, 96 \%$, and $92 \%$, respectively) were able to do so. Responses included: "I would get pins in my bone" or "they will also put in a bar". 


\section{Generally good}

For the question "Can you think of any good things about being in the study", only $56 \%$ of the youngest children were able to give a reasonable response compared with $75 \%$ of the $10-12$ year olds, $82 \%$ of the $13-15$ year olds, and $76 \%$ of the adults. This difference was significant $\left(X^{2} \quad(3)=21.4\right.$, $\mathrm{p}<0.01$ ). Reasonable responses included "helping doctors find the best method", "helping others", or "learning things".

\section{Generally bad}

In response to the question "Can you think of any bad things about being in the study?" a significant age effect was found $\left(X^{2}(3)=21.5, p<0.001\right)$, where $64 \%$ of the youngest children gave an appropriate answer, compared with $80 \%$ of the middle children, $76 \%$ of the oldest children, and $86 \%$ of the adults. Reasonable responses included "my leg may not heal well" or "it would hurt".

\section{General questions}

When asked: "will everyone get the same operation?" Almost all participants, regardless of age group, correctly answered "No" $(91 \%, 89 \%, 92 \%$, and 92\%, for each age group respectively). Most of the participants had difficulty understanding that they would not get to choose the operation, should they agree to participate in the study: a significant age effect was found, where $55 \%$ of the youngest participants answered this question correctly, compared with $23 \%$ of the middle, $28 \%$ of the oldest, and $36 \%$ of the adults $\left(X^{2}(9)=32.3, \underline{p}<0.0001\right)$. When asked if they would agree to be in the study, the majority of participants in all four age categories said yes $(62 \%, 75 \%, 79 \%$, and $80 \%$, respectively). The age effect was significant: $X^{2}(6)=20.5, p<0.0022$. When asked what would happen if they decided not to be in the study, a significant age effect was found, where only $64 \%$ of the youngest children understood that they would still get the standard cast operation compared with participants in the other three categories (79\%, 85\%, and $86 \%$, respectively), $\mathrm{X}^{2}(12)=31.6, \mathrm{p}<0.001$.

Finally, participants were asked if they had ever had a broken bone: $17 \%$ of the $6-9$ year olds, $30 \%$ of the $10-12$ year olds, and $34 \%$ of the $13-15$ year olds answered affirmatively. Thirty one per cent of the adults had experienced a broken bone.

\section{Risks and benefits}

The risks and benefits varied as a function of which condition the participant had been assigned to. Table 1 displays the choice of operation as a function of age category and condition. The correct choice, based on the good and bad things mentioned, is highlighted for each condition.

It is clear from the table that overall, younger subjects preferred the cast to the pins; however most children, regardless of age group, were able to choose the "correct" option, based on an analysis of the balance of good and bad things associated with each procedure. In the HHHL condition ("high" good and "high" bad things about the cast, "high" good and "low" bad things for the pins) the correct choice would be the pins procedure. The youngest children in this condition chose the pins slightly more often than the cast, but significantly less often than children in the other two age groups $(\underline{p}<0.005)$. Interestingly, in one condition (LHLH) that indicated "low" good things for both procedures and "high" bad things for both-therefore an "even" choice-the children in the younger two categories were significantly more likely to choose the cast, whereas the oldest were almost evenly divided in their decisions $(\mathrm{p}<0.001)$.
Table 1 Choice of operation (\% cast $v \%$ pins) as a function of age category and condition

\begin{tabular}{llllll}
\hline \multicolumn{5}{c}{ Age group } \\
\cline { 3 - 6 } Condition & Operation & $6-9$ & $10-12$ & $13+$ & Parents \\
\hline Participants & & $(82)$ & $(89)$ & $180)$ & $(237)$ \\
All Conditions & Cast & $68 \%$ & $58 \%$ & $44 \%$ & $31 \%$ \\
(p $<0.0000)$ & Pins & $28 \%$ & $35 \%$ & $51 \%$ & $59 \%$ \\
HHLH & Cast & 83 & 67 & 75 & 51 \\
(ns) & $\overline{\text { Pins }}$ & 17 & 33 & 25 & 49 \\
HLLL & Cast & 87 & 83 & 64 & 69 \\
(ns) & $\overline{\text { Pins }}$ & 13 & 17 & 36 & 31 \\
LHLL & Cast & 57 & 23 & 29 & 15 \\
(ns) & Pins & 43 & 77 & 71 & 85 \\
HHHL & $\overline{\text { Cast }}$ & 46 & 38 & 11 & 0 \\
(p $<0.005)$ & Pins & 54 & 62 & 89 & 100 \\
LHLH & $\overline{\text { Cast }}$ & 83 & 94 & 42 & 25 \\
(p $<0.001)$ & Pins & 17 & 6 & 58 & 75 \\
HLHL & $\overline{\text { Cast }}$ & 67 & 63 & 46 & 45 \\
(ns) & Pins & 33 & 37 & 54 & 55 \\
\hline
\end{tabular}

The ordering of the letters for each condition indicates, in order, the high and low risks and benefits associated with each operation. $\mathrm{HHLH}-$ for example, refers to high benefits and high risks associated with the cast operation and low benefits and high risks associated with the pins operation. The "correct" choice, based on the balance of risks and benefits is highlighted in bold for each condition.

\section{DISCUSSION}

The results of this study support our hypothesis that even very young children can understand potentially difficult and complex concepts such as the risks and benefits associated with their participation in biomedical research. We have examined each of the components of the information process.

\section{Research}

This particular section of the information form was not well understood by the children in the two youngest groups. Although it may be the case that the youngest children were, because of developmental issues, unable to understand this concept, the results from the other sections of the paper suggest that it may instead be due to the complexity of the information. The fact that the majority of the older children as well as the adults were able to understand this section suggests that, rather than the concepts being too difficult to understand, the forms need to be rewritten in more simplified language. This was the first time we had included such a section in one of our information forms so it had not been previously extensively tested.

\section{Purpose}

The purpose of the studies appeared to be well understood by participants of all ages, including the youngest children. In a pilot study that had been completed before the start of the study described in this manuscript, the purpose had not been well understood by children of all ages. Following the pilot study, we had amended the wording of the "purpose". It is encouraging to observe that simplifying the wording improved comprehension.

\section{Procedures}

These sections of the forms also appeared to be well written for each of the age groups, independent of whether they were asked about general procedures or procedures specific to the particular treatment options. The majority of all participants were able to understand the information presented to them. These findings imply that even complicated procedures, involving multiple steps, can be explained to all of the age groups from whom assent would be requested. 


\section{General questions}

The results for this section were excellent; almost every participant understood that not all participants would get the same operation. The component that was not as well understood was the concept of random assignment. Many subjects did not understand that to minimise bias, it was necessary to choose the operation by a random method-for example, by the flip of a coin, rather than leaving the choice up to the individual. It is not clear why the youngest participants correctly indicated they would not have a choice more often than participants in the other conditionsperhaps, given that even older participants had difficulty understanding this aspect, the younger children were more likely to try to guess the correct choice, and so were right about half the time. This should be examined further in future studies. Nevertheless, almost all participants indicated they would be willing to take part in such a study if they were in the situation described in the information forms. Such a finding should be encouraging to those who are concerned that, by explaining research in detail to individuals (especially children), they will lose potential research participants.

\section{Risks and benefits}

In general, the younger participants chose the cast operation most often, whereas the older participants were more likely to choose the pins procedure. This trend continued, even when there was a "correct" choice associated with a particular condition. Among participants in the "cast correct" conditions, the majority chose the correct option. Whereas the majority of the youngest children chose the cast, adults were still less likely to endorse such an option. When the pins operation was the correct choice, the youngest children were split as to which operation they would choose, whereas the oldest children and the adults overwhelmingly endorsed the correct option. When the choice was ambiguous, the findings were similar to the overall results: the younger children invariably chose the cast, while the older participants were more willing to try the new pins procedure. These results suggest that younger children are more likely to choose the option with which they are most familiar-that is, a cast, despite risks that might outweigh benefits. Older children and adults pay more attention to the advantages and disadvantages of either option-for example, the greater mobility afforded by the pins procedure. The implication for those writing information forms targeted at young children is that they must ensure all the risks and benefits are clearly and simply outlined so that an informed choice can be made.

The study was not without limitations; it was quite difficult to come up with balanced "good and bad things" associated with each of the six conditions that would have equal appeal to all age groups. As was observed with the younger children tending to choose the familiar cast operation most often, the weighing of the risks and benefits seems to have been age dependent. It is difficult to determine the generalisability of the results of this study. It is possible that the levels of comprehension and understanding demonstrated by our sample would be different (higher or lower) if children who really had just broken their thigh bones had been recruited for the study.

Generally, the results of this study suggest that all individuals, regardless of age, can understand most of the differences between two complicated treatment options, and the associated risks and benefits. Therefore they can benefit from carefully worded information forms. This lends support to the idea that some of the variability in levels of understanding reported in previous studies may have been a function of how the assent forms were written as opposed to a developmental barrier that children under nine years of age had difficulty crossing. It is also important to note that participants seemed to base their choices, at least in part, on more than just the "facts". Therefore decisions to participate in research may involve two processes: a basic understanding of the facts, as well as a personal level of comfort with the choices. It may be that an understanding of the former is required before the latter can be evaluated.

While assent itself is not sufficient for participation in research there are interesting implications should the child disagree once the parent consents; as noted by Vitiello, ${ }^{18}$ if the child is adamant that she does not wish to continue, this wish should be respected. He further notes, however, that it is imperative that parents and researchers work closely in these situations in order to better understand the feelings of the child. Although legally the parent still has the option to override the child's wishes, ethical concerns may remain. Although beyond the scope of the present study, this should perhaps be examined in the future.

The results of this research protocol support the notion that even young children, if presented with information in an age appropriate format, can understand and respond appropriately to the process of assent. Although this is not the same as suggesting that young children can ultimately consent to the research, it does mean that they can, and should, be informed and consulted as part of the overall process. Having completed this project using "surrogate" patients, we are now ready to test these forms on children in the "real world"-that is, on children actually experiencing, or suffering from, the disease or procedure described in these research forms. One may imagine that these "experienced" children may show even greater understanding than those who completed this study. The current results suggest that it is indeed worth the effort to create meaningful assent forms for even very young children: their ability to understand complex information should not be underestimated.

\section{Authors' affiliations \\ T M Burke, Department of Psychology, Ryerson University, Toronto, Ontario, Canada \\ R Abramovitch, Department of Psychology, University of Toronto, Toronto, Ontario, Canada \\ S Zlotkin, Departments of Paediatrics and Nutritional Sciences, University of Toronto, Hospital for Sick Children, Toronto, Ontario, Canada}

This research was supported by a research grant from the Hospital for Sick Children Foundation to the second and third authors.

\section{APPENDIX A}

\section{PRETEND STUDY}

Information sheet (HHHL)

This is a research study that compares two ways to fix a broken leg_using a cast or using pins.

\section{What is research?}

Doctors and nurses know a lot about how to find out what is wrong with you when you are sick and how to make you better. But, there are still some things that we don't know but would like to find out about. The way we learn more about how to make kids better is by doing research. Research is trying new and different ways of making kids better, and asking questions about how they feel.

\section{Why are we doing this study?}

Pretend you have a broken bone in your thigh (just above your knee) which will not get better by itself. You need an operation to make it better. There are two different kinds of operations that we could use to fix your leg. One is an old operation and one is a new operation. We already know that both of these operations work, but they might have different 


\section{Good things about the study}

About the cast
1. The cast operation works on
almost everyone. Only about
$5 \%$, or five people out of every
hundred, need to have another
operation.

2. After the cast operation you can go home in two or three days.

\section{About the pins}

1. The pins operation works on almost everyone. Just like with the cast operation, only about five people out of every hundred need to have another operation.

2. Just like with the cast operation, you can go home in two or three days after having the pins put in

3. There will only be two small cuts where they fix your leg, so the scar will be almost invisible.

3. Because the pins are so small, the marks on your leg will be almost invisible.
Bad things about the study

\begin{tabular}{ll}
\hline About the cast & About the pins \\
\hline $\begin{array}{l}\text { 1. There is some chance that you } \\
\text { will get a serious infection with } \\
\text { the cast. About } 25 \% \text {, or one out } \\
\text { of every four people who have } \\
\text { the cast, will get an infection. }\end{array}$ & $\begin{array}{l}\text { 1. There is not much chance that get a serious infection. } \\
\text { of every } 100, \text { will get one. }\end{array}$ \\
$\begin{array}{ll}\text { 2. Sometimes damage to the } \\
\text { nerves in your legs can occur }\end{array}$ & $\begin{array}{l}\text { 2. Damage to the nerves in your } \\
\text { legs can occur with the pins } \\
\text { operation, but the chance of this is } \\
\text { smaller than with the cast. }\end{array}$ \\
$\begin{array}{ll}\text { 3. It is hard to move with the } \\
\text { cast on, even with the help of } \\
\text { crutches. }\end{array}$ & $\begin{array}{l}\text { 3. You will not be able to move } \\
\text { around much for the first few days, } \\
\text { but then you can start moving after } \\
\text { that. }\end{array}$ \\
\hline
\end{tabular}

good things and bad things about them. We want to know which of these two ways is best for a person with a broken leg. We want to see if there are differences in how the leg heals and how long it takes for kids to be able to walk around without any problem. We also want to know how much kids like or don't like each of the operations.

\section{What will happen in the study?}

If you decide that you will be part of our study you will get an operation on your broken leg. We will decide which operation you will get by chance-just like flipping a coin or pulling numbers out of a hat. If you do not want to be part of this study, you will still have an operation, only it will be the cast operation for sure, not the pins.

Some things will be the same for both operations and some things will be different. With both operations we will give you some medicine to make you go to sleep for a little while so that you won't feel any pain while we are fixing your leg.

\section{If you have the "cast" operation}

We will fix your broken leg bones by putting you in something called a "body" cast, which means that the cast will go from your chest to your toes. This will help to keep your bones together so that they can heal properly. The cast will have to stay on for 6 to 12 weeks, but you will have crutches to help you move around. This is the operation we usually do at the Hospital for Sick Children.

\section{If you have the "pins" operation}

We will fix your broken leg bones by using pins to help keep the bones together. We will put a few pins in your thigh starting just above your knee and going the rest of the way up your leg. You will be able to see the pins because they will go from the outside of your leg to the inside of the bones in your leg. We will make sure that the pins keep your leg bones straight by connecting them with a long bar on the outside of your leg. The pins and the bar have to stay on for about 6 to 12 weeks, but you do not need a cast. You will be able to move around without crutches. This is the new operation that we want to compare to the old one.

For both of the operations, whether you have the cast or the pins, a nurse will come every week to make sure that everything is okay. Someone will also be calling you and your parents on the telephone to ask questions about how you are doing. You will have to come back to the hospital once every week for an $x$ ray of your leg so that the doctors can see how your bone is healing. Once the bone has healed, you will have to come back to the hospital for $x$ rays four more times-at 3 , 6,9 , and 24 months. That means that the doctors will keep seeing you for another two years. The doctors will also check to see if you can move and use your leg without any problems. These tests will tell us if the operation has worked and help us make sure that there are no problems with it.

Either of these operations will help your broken leg bone get better, but there are good and bad things about both of them. By doing this research we should be able to tell if one operation is better than the other.

Remember, if you do not want to be part of this study, you will still have an operation, only it will be the cast operation for sure, not the pins.

\section{REFERENCES}

1 Green JB, Duncan RE, Barnes GL, et al. Putting the "informed" into "consent": A matter of plain language. J Paediatr Child Heath 2003;39:700-3.

2 Medical Research Council of Canada. Guidelines on research involving human subjects. Ottawa: Minister of Supplies and Services, 1987.

3 National Council on Bioethics in Human Research Consent Panel Task Force. Revised report on research involving children. Ottawa: National Council of Bioethics in Human Research, 1993.

4 US Department of Health and Human Services. Protection of human subjects, OPRR Reports. Washington, DC: US Government Printing Office, 1991 (title 45 CFR 46 subpart B, Revised June 18, 1991).

5 Spriggs $M$. Canaries in the mines: children, risk, non-therapeutic research, and justice. J Med Ethics 2004;30:176-81

6 Weithorn L, Campbell S. The competency of children and adolescents to make informed treatment decisions. Child Development 1982;17:1589-98.

7 Lewis C, Lewis M, Ifekwunigue M. Informed consent by children and participation in an influenza vaccine trial. Am J Public Health 1978;68:1079-82.

8 Susman EJ, Dorn LD, Fletcher JC. Participation in biomedical research: the consent process as viewed by children, adolescents, young adults, and physicians. J Pediatr 1992;121:547-52.

9 Abramovitch R, Freedman J, Thoden K, et al. Children's capacity to consent to participation in psychological research: empirical findings. Child Development 1991;62:1100-9.

10 Ondrusek N, Abramovitch R, Pencharz P, et al. Empirical examination of the ability of children to consent to clinical research. J Med Ethics 1998;24:158-65

11 Cassileth BR, Zupkis RV, Sutton-Smith K, et al. Informed consent-why are its goals imperfectly realised? N Engl J Med 1980;302:896-900.

12 Schultz AL, Pardee GP, Ensinck JW. Are research subjects really informed? West J Med 1975;123:76-80.

13 Priluck IA, Robertson DM, Buettner $\mathrm{H}$. What patients recall of the preoperative discussion after retinal detachment surgery. Am J Ophthalmol 1979:87:620-3.

14 Silva MC, Sorrel JM. Enhancing comprehension of information for informed consent: a review of empirical research. IRB 1988;10:1-5.

15 Abramovitch R, Freedman J, Henry K, et al. Children's capacity to agree to psychological research: knowledge of risks and benefits and voluntariness. Ethics Behav 1995:5:25-48.

16 Ogloff JRP, Otto RK. Are research participants truly informed? Readability of informed consent forms used in research. Ethics Behav 1991;1:239-52.

17 Epstein L, Lasagna L. Obtaining informed consent: form or substance. Arch Int Med 1969;123:682-8.

18 Vitiello B. Ethical considerations in psychopharmacological research involving children and adolescents. Psychopharmacology 2003;171:86-91. 